\title{
THE CONTENT KNOWLEDGE OF TURKISH AND SLOVAK PRE-SERVICE ELEMENTARY TEACHERS: THE CASE OF THE SQUARE Asuman Duatepe-Paksu ${ }^{1}$, Katarína Žilková ${ }^{2}$
}

\begin{abstract}
This study compares the content knowledge of Slovak and Turkish pre-service teachers regarding the classification of the square. The study participants were final year students of training departments for elementary teachers. These pre-service teachers had completed all obligatory geometry courses in their program. For data collection, six questions leading to 10 items for analysis were used in this study. The total correct responses for each item were calculated as percentages for the Slovak and Turkish groups each and a chi-square test of independence was performed to see how well pre-service teachers understood the square in terms of quadrilateral relationships and whether groups of pre-service teachers from different countries displayed significantly different scores. According to the results, the responses of the Turkish and Slovak pre-service teachers were statistically different on each item. Slovak pre-service teachers displayed a significantly higher score on items relating to the class inclusion of a square with a rectangle, a parallelogram, and a quadrilateral. Turkish pre-service teachers presented a significantly higher score for items questioning the class inclusion of a square with a rhombus and a polygon.
\end{abstract}

UDC Classification: 37; DOI: http://dx.doi.org/10.12955/cbup.v6.1213

Keywords: pre-service teacher, content knowledge, square, quadrilaterals

\section{Introduction}

Teachers' content knowledge pertains to an understanding of a subject and its organizing structures (Shulman, 1986). Many educators would not argue about the significance of such knowledge in the field of teaching. Notwithstanding its importance, when it comes to geometry content knowledge with a focus on quadrilaterals, the literature reveals that pre-service and in-service teachers have problems with classifications (Duatepe-Paksu et al., 2012; Duatepe-Paksu et al., 2017; Fujita \& Jones, 2006; Günhan, 2014; Pickreign, 2007; Rianasari et al., 2016; Žilková et al., 2015). While Duatepe-Paksu et al. (2012; 2017) worked on pre-service teachers' content knowledge, specifically rhombus, and Pickreign, (2007) on rhombus and rectangle, other studies worked on various quadrilaterals.

Rianasari et al. (2016) investigated Indonesian primary teachers' understanding of inclusion relationships of quadrilaterals. According to their report, only $36 \%$ of the teachers identified the class inclusion relationship between square and rectangle based on acknowledging valid conditions: because a square has equal sides and four right angles; because a square has right angles and the opposite sides are equal; and because it has right angles and two pairs of parallel sides.

Fujita and Jones (2006) analyzed Scottish pre-service primary teachers for their geometry content knowledge on defining and classifying quadrilaterals. They reported that $12.7 \%$ of the pre-service teachers answered the question correctly on whether a square is a rectangle. In their study, the preservice teachers were given quadrilaterals and asked to draw arrows to identify the relationship between particular pairs of quadrilaterals to indicate when one shape was a special case of the other. On one hand, $65 \%$ of participants regarded a square as a special case of a rectangle and $40 \%$ of participants accepted a square as a special case of a rhombus. On the other hand, $21 \%$ considered a rectangle as a special case of a square, while a similar number of participants regarded a rhombus as a special case of a square.

Türnüklü et al. (2013) also examined the inclusion relationship between a square and a rectangle. In their study, only one (of nine) Turkish mathematics teacher could state the correct relationship, i.e., that a square is a special kind of a rectangle.

By considering these studies, we aim to analyze and compare the content knowledge of elementary preservice teachers on the class inclusion of the square with other geometric shapes. Namely, we consider a rectangle, a rhombus, a parallelogram, a quadrilateral, and polygon and their inclusion relationship with a square. The square was chosen because of its familiar use throughout school mathematics curriculum. Figure 1 shows the hierarchical classification considered in this study.

\footnotetext{
${ }^{1}$ Pamukkale University, Turkey; aduatepe@pau.edu.tr

${ }^{2}$ Comenius University in Bratislava, Slovakia; katarina@zilka.sk
} 


Figure 1: Hierarchical classification considered in the study

\section{Data and Methodology}

The participants of this study were final-year students of elementary teachers from the teacher training departments of two Slovak and one Turkish university. A total of 144 pre-service primary teachers participated in the study (25 from the Catholic University, Ružomberok, 59 from the Comenius University, Bratislava, and 60 from the Pamukkale University, Turkey). These pre-service teachers had completed all compulsory geometry courses in their program. Thus, they were expected to understand the geometry content knowledge as offered in their teacher training program.

To collect data, several items of the polygon and quadrilateral test, constructed by Žilková (2013), were used. The original research tool was a test with multiple-choice questions (one or more than one answers could be correct). Six questions were composed covering 10 items taken from this test. Two items (5a and 10) were about class inclusion between a square and a rhombus. Three items (5d, 15, and 16) covered class inclusion between a square and a rectangle. Two ( $5 \mathrm{c}$ and 9$)$ were related to class inclusion between a square and a parallelogram, and one (5b) was on class inclusion between a square and a quadrilateral while the remaining two items $(D, R)$ dealt with class inclusion between square and polygon. The questions and their aims are displayed in Table 1.

Percentages were calculated and a chi-square test of independence was used to examine the differences between Slovak and Turkish student responses for each item.

\section{Results and Discussion}

The total of correct responses as percentages and the result of a chi-square test of independence between Slovak and Turkish groups are displayed in Table 2. Data analyses showed significant differences between both study groups on each item $(\alpha=0.05)$. While Slovak pre-service teachers achieved a higher percentage of success in items $5 \mathrm{~b}, 5 \mathrm{c}, 5 \mathrm{~d}, 9,15$, and 16 , the Turkish pre-service group achieved a significantly higher percentage in items $5 \mathrm{a}, 10, \mathrm{D}$, and $\mathrm{R}$.

The pre-service teachers' understanding of the relationship between the class of a square and a rectangle involved analyzing items 15,16 , and $5 \mathrm{~d}$. The differences between items involved their formulation. Both, items 15 and 16 examined the ability of participants' understanding of logical implications about the statements: 'a square is always a rectangle' and 'a rectangle is sometimes a square'.

Understanding of Relationships between Squares and Rectangles

There were no observed differences between the Slovak and Turkish responses in terms of introduction terminology for these concepts. Nevertheless, results showed statistically significant differences between the Slovak and Turkish study groups for the results shown in Table 1. Almost all Slovak students responded that a square is always a rectangle (93\%), indicating their awareness of the inclusion relationship between a square and a rectangle. However, for item 16, their success rate was only 58\%, which could mean that the change in wording and formulation of the question caused a decline in their understanding. In other words, determining the answer for the 'opposite' direction of the relationship between a rectangle and a square was more difficult for them. Hence, the reason for this result might not be their unfamiliarity with properties of a square and a rectangle. Moreover, a similar difference in the success rate of these items was observed in the research group of Turkish teacher candidates (item 15 with $63 \%$; item 16 with $21 \%$ ). We assume that the problem was caused by the formulation of question 16 with linguistics playing a major role. Nonetheless, a successful outcome ensues from understanding and correctly interpreting the necessary and sufficient conditions. 


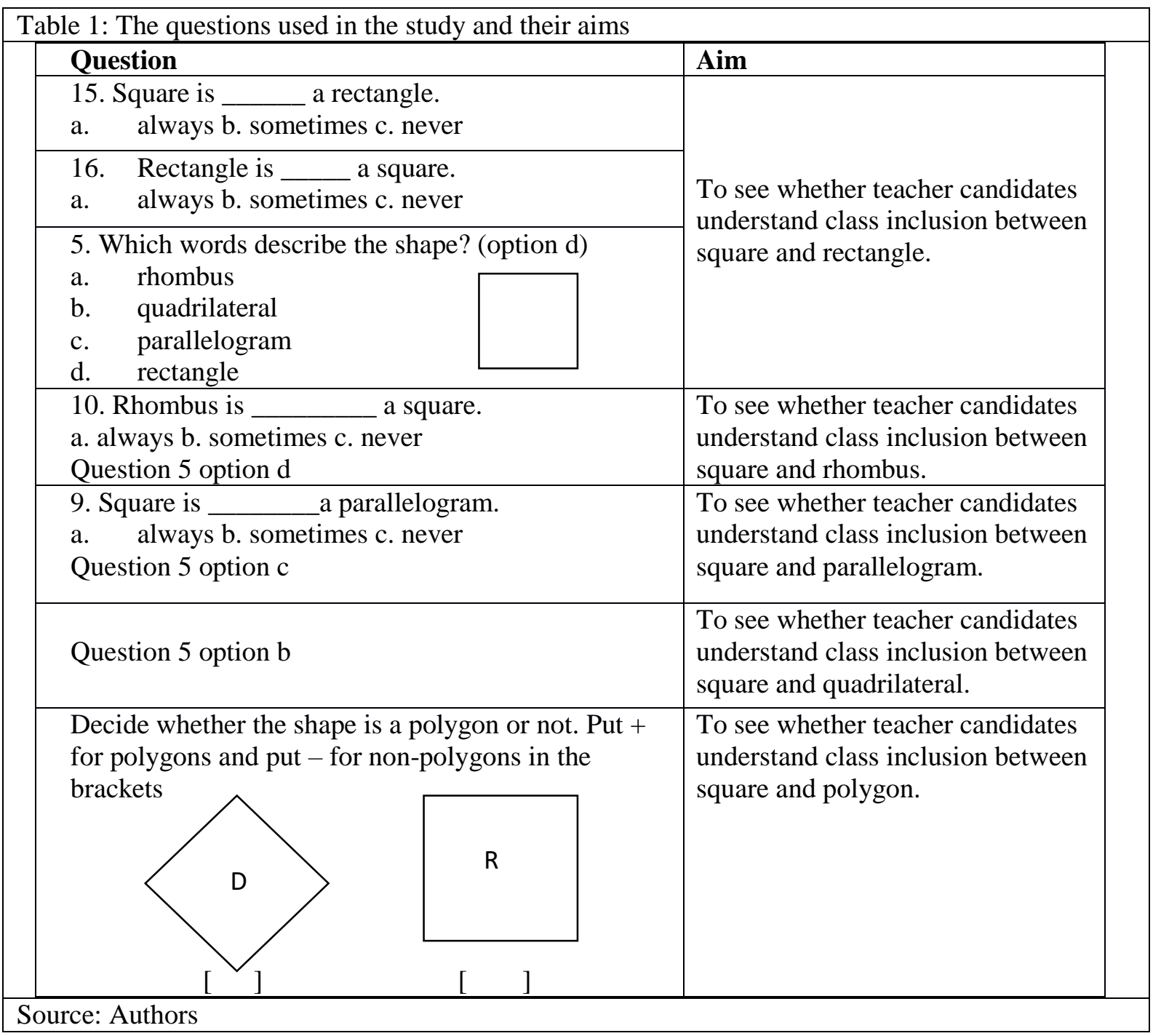

Table 2: The correct response percentage of groups and results of chi-square test of independence

\begin{tabular}{|l|r|r|r|r|}
\hline Item & \multicolumn{1}{|l|}{ SVK } & \multicolumn{1}{|l|}{ TUR } & \multicolumn{1}{l|}{$\boldsymbol{\chi}^{\mathbf{2}} \mathbf{( 1 )}$} & p-value \\
\hline $\mathbf{5 a}$ & 4 & 57 & 51,680 & $<.001$ \\
\hline $\mathbf{5 b}$ & 91 & 42 & 47,868 & $<.001$ \\
\hline $\mathbf{5 c}$ & 75 & 17 & 47,648 & $<.001$ \\
\hline $\mathbf{5 d}$ & 87 & 13 & 76,981 & $<.001$ \\
\hline $\mathbf{9}$ & 81 & 63 & 6,486 &, 011 \\
\hline $\mathbf{1 0}$ & 20 & 40 & 6,712 &, 010 \\
\hline $\mathbf{1 5}$ & 93 & 63 & 19,477 & $<.001$ \\
\hline $\mathbf{1 6}$ & 58 & 21 & 19,192 & $<.001$ \\
\hline $\mathbf{D}$ & 76 & 97 & 10,864 &, 001 \\
\hline $\mathbf{R}$ & 76 & 95 & 8,780 &, 003 \\
\hline
\end{tabular}

(SVK: Slovak, TUR: Turkish) Source: Authors

Apart from questions 15 and 16, question 5 also had an item examining the class inclusion between a square and a rectangle. In question 5 (Table 1), a square was given and pre-service teachers were asked to select an option(s) to describe the shape. All options, namely, rhombus (5a), quadrilateral (5b), parallelogram (5c), and rectangle (5d), could describe the shape given. A high percentage of the Slovak students correctly answered with a square as a rectangle (87\%) by choosing $5 \mathrm{~d}$. A comparison of the differences between items 15 and $5 \mathrm{~d}$ revealed the students were more successful with item 15 , despite both items examining the same inclusion relationship. This difference was not significant within the results of the Slovak study group (6\% success rate). However, the results of the Turkish participants 
showed a significant difference between the success rate of items 15 (63\%) and 5d (13\%). In other words, while $63 \%$ of the Turkish students responded with a square as always being a rectangle, when they were given a square only, $13 \%$ of them selected the option of the rectangle to describe that shape. This interesting finding may be interpreted by considering all answers to question 5 ( $5 \mathrm{a}, 5 \mathrm{~b}, 5 \mathrm{c}$, and $5 \mathrm{~d}$ ) as shown in Table 3 . When they were given a square, the Turkish group mostly selected 'rhombus' (5a) to describe it. Only $1 \%$ selected all options simultaneously. This could be interpreted as the Turkish group was inclined to choose only one option. They might select the first possible answer (i.e., option 5a) and then move on to the next question.

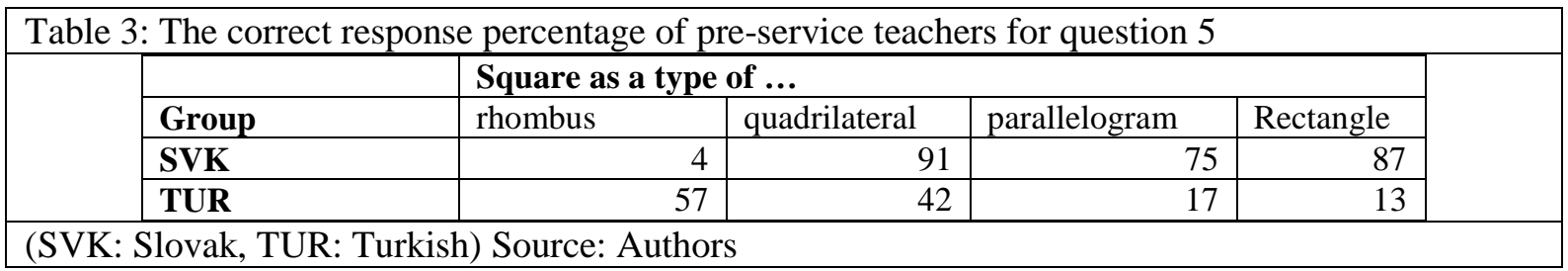

Understanding of Relations between Square and Other Upper Classes Shape

As explained above, question 5 with 4 items aimed to determine class inclusion relationships other than the relationship between square and rectangle. The goal of the other items was to determine whether students perceived the class of a square as a subset of quadrilateral, parallelogram, and rhombus classes. The results of the analysis showed statistically significant differences between the group of Slovak and the group of Turkish students for question 5 (Table 2).

The Slovak group had significantly better results for items querying relationships of a square and a quadrilateral, a square and a rectangle, and a square and a parallelogram. The group was better at identifying a square as a quadrilateral, which is the overarching class among the options. The second trouble-free class inclusion for the Slovak group was an inclusion relationship between a square and a rectangle. Three-quarters of Slovak students identified a square in the diagram as a parallelogram. In contrast, it was evident that the Slovak students were unable to perceive a square as a subset of a rhombus. The details of the analyses for these items are explained in the following parts.

Understanding of Class Inclusion between Squares and Rhombus

The results of data analyses for item 5a and 10 display pre-service teachers' comprehension of class inclusion between square and rhombus. Values from Table 2 indicate that the group of Turkish students reached a significantly better result in understanding the relationship between a square and a rhombus. For item 5a, with the correct response percentage of 57\%, this group regarded the model of the given square as a type of rhombus. In comparison, the correct response percentage of Slovak group about this relationship (a square and a rhombus) was only at the level of 4\%. This result is not surprising considering the way the terms, square and rhombus, are defined and introduced in Slovak terminology. Exclusive introduction of the term rhombus (as a parallelogram with all equal sides in length, which is not a square) determines an understanding of the class of a square and that of a rhombus, disjunctively. However, the group of students in Slovakia consisted of only those who completed courses about the introduction of quadrilaterals according to the inclusion approach. Therefore, the students of Slovak were exposed to the inclusion introduction of terms. However, they either preferred the definition according to the Slovak terminology they had learned beforehand or they were not able to understand inclusion relationships between quadrilaterals. Therefore, we deduce that the Slovak students considered a square as a shape that is a subset of quadrilateral, rectangle, and parallelogram but they are not able to consider the inclusion of a square in the class of a rhombus.

The analysis of results of item 10 indicates that Slovak students failed to understand inclusion relationships between a square and a rhombus. This was a word question and pre-service teachers needed to decide whether a 'Rhombus is (a) always, (b) sometimes, or (c) never a square.' In other words, they needed to know the class inclusion and make a logical deduction about this relation to fill the blank correctly. Only $20 \%$ of Slovak students chose the correct answer that a rhombus is sometimes a square. In comparing the results of items on the relationship between a square and a rhombus (5a and 10), the Slovak students displayed the lowest score in both items. Therefore, these items appear the most difficult for them as many participants were unable to give the correct answer. The result is understandable when considering the educational approach to quadrilaterals in Slovakia. This means that the manner in which 
terminology is introduced affects the pre-service teachers' enduring view of the properties of different types of quadrilaterals, i.e., regardless of further training.

For these items, the correct response rate of the Turkish group was $40 \%$. Although the Turkish group displayed a significantly better score then the Slovak group, the figures showed that less than half of the Turkish group responded that a rhombus can be sometimes a square (when all angles of the form are equal or at right angles). Table 4 displays the percentage for each of the three options as selected by the groups. As shown in the table, the Turkish group displayed a higher percentage of pre-service teachers responding with 'a rhombus is always a square' than the correct answer of 'sometimes'.

Table 4: The correct response percentage of pre-service teachers for question 10

\begin{tabular}{|c|c|c|c|}
\hline & Options & & \\
\hline Group & always & sometimes & never \\
\hline SVK & 31 & 20 & 49 \\
\hline TUR & 48 & 40 & 11 \\
\hline
\end{tabular}

(SVK: Slovak, TUR: Turkish) Source: Authors

Understanding of Class Inclusion between Square and Parallelogram

The results of data analyses for items $5 \mathrm{c}$ and 9 indicated the pre-service teachers' comprehension of class inclusion between a square and a parallelogram. Question 9 was formed using words without diagrams. To be successful in this item, the participants needed to understand, not only the quantifiers of always, sometimes, and never, but also the logical deduction about the necessary and sufficient conditions. The formulation of the item $5 \mathrm{c}$ was similar to that of item 9 although they differed formally. In the formulation of $5 \mathrm{c}$, a diagram of a square was given and the item was to decide whether the diagram was a model of a parallelogram. The results showed that the Slovak students perceived a square as a type of a parallelogram at the level of $81 \%$, which is a mildly higher success rate than that resulting for item $5 \mathrm{c}(75 \%)$. As these two results (items $5 \mathrm{c}$ and 9 ) corresponded, they showed that up to approximately four-fifths of the students within the Slovak group recognized that a square is a subset of a parallelogram. This indicates that a large portion of the Slovak group understood that all properties of a parallelogram are also properties of a square.

Remarkably, the results of the Turkish group for items $5 \mathrm{c}$ and 9 appear inconsistent (Table 2). The difference between the correct response percentage for item 9 is almost four times that of item $5 \mathrm{c}$ (17\% for $5 \mathrm{c}$ and $63 \%$ for 9). Parallel to the result for the Turkish group on the class inclusion between a rectangle and a square, this finding could be interpreted as not all options of question 5 were considered (Table 3). That is, most of the Turkish group selected 'rhombus' (5a) to describe the square and then made no effort to consider and then select other option(s).

Understanding of Class Inclusion between Squares and Polygons

The general classification of a square into the class of polygons was mostly observed in items D and R. The aim of these items was to examine whether teacher candidates considered a square as a subset of a polygon. Almost all Turkish pre-service teachers gave the correct response to items D and R $(97 \%$ and $95 \%$, respectively), which indicated that they regarded a square as a subset class of the polygon. In comparison, the Slovak pre-service teachers marked shapes in diagrams as polygons at the level of 76\%, which was displayed for both $\mathrm{D}$ and $\mathrm{R}$. This result indicates that approximately a quarter of the Slovak group did not perceive the squares in the diagram as polygons. An interview of some teacher candidates after the test to investigate why they failed to recognize a square as a polygon revealed the following. Their most common answer was that triangles and quadrilaterals are not polygons, but pentagons and hexagons are polygons. One potential interpretation is that the Slovak mathematical education attributes special attention to triangles and quadrilaterals more so than other polygons, which are taught later in a separate chapter about polygons (in lower secondary education). In other words, triangles and quadrilaterals are taught separately and the latter is usually taught without connection to polygons. Another possible interpretation is a linguistical one. The word 'poly' meaning 'many' could cause the students to reason that a polygon has many sides and many angles (more than four possibly). Their judgments on three- and four-sided shapes may be that there is an insufficient number of sides, and some may reason that five sides may be insufficient. Nonetheless, the results on the understanding of a square as a polygon for the Slovak study group signify that some students have uncertainties about classifying a square as a polygon. 


\section{Conclusion}

This study revealed that the responses of the Turkish and Slovak pre-service teachers were statistically different for every item of the test. A significantly higher percentage of Slovak compared to Turkish pre-service teachers answered correctly for the items relating to the class inclusion of a square as a rectangle, a parallelogram, and a quadrilateral. In contrast, a significantly higher percentage of Turkish compared to Slovak pre-service teachers answered correctly for the items questioning class inclusion between a square and a rhombus and polygon. More important, the major result of this study was the pre-service teachers' correct response level for each of the items. Questions used in the study were at a basic level so that even middle grades students could correctly answer them. However, findings revealed that except for two items (5b and 15) for the Slovak group and two items (D and R) for the Turkish group, participants correct response rate was below $90 \%$.

This finding is parallel with that of the research where Indonesian primary teachers, the Scottish preservice primary teachers, and Turkish mathematics teachers reportedly had problems with class inclusion relating with square (Rianasari, et al, 2016; Fujita and Jones, 2006; Türnüklü et al, 2013; respectively).

The poor results found with word questions could have a formal and linguistic basis. The necessary deductive reasoning was not achieved by most of the participants. It can be concluded that participants did not operate in the second van Hiele Geometric thinking level (1986) as participants at the expected level of comprehending in class inclusion.

These results need to be considered in line with the limitations of the study. The study sample of students was chosen based on convenience. It is recommended that the results be verified with future studies using more broad and representative groups. Cultural factors, such as language, differences in education approaches in geometry teaching, and differences between national terminologies could have affected results. More research involving various student groups is recommended to examine other cultures.

\section{Acknowledgment}

This study was supported by 2018KRM002-162 (2018KRM002) ADEP 2018.

\section{References}

Duatepe-Paksu, A., Rybanský, L. \& Žilková, K. (2017). The Content Knowledge about Rhombus of Turkish and Slovak PreService Elementary Teachers. Proceedings of SEMT'17, 158- 168

Duatepe-Paksu, A., Pakmak, G. S., \& İymen, E. (2012). Pre-service Elementary Teachers' Identification of Necessary and Sufficient Conditions for a Rhombus. Procedia Social and Behavioral Sciences, 46, 3249-3253.

Günhan, B. C. (2014). An Investigation of Pre-Service Elementary School Teachers' Knowledge Concerning Quadrilaterals. Çukurova University. Faculty of Education Journal, 43(2), 137-154.

Fujita, T., \& Jones, K. (2006) Primary trainee teachers' understanding of basic geometrical figures in Scotland. Proceedings of the 30th Conference of the International Group for the Psychology of Mathematics Education, 3, 14-21.

Pickreign, J. (2007). Rectangles and Rhombi: How Well Do Pre-service Teachers Know Them? IUMPST The Journal, 1, 1-7

Rianasari, V. F., Hongki, J. \& Niluh, S. (2016). Primary teachers' understanding of the inclusion relations of quadrilaterals. Proceeding 2nd International Conference on Education and Training. 263- 265.

Shulman, L. S. (1986). Those who understand: Knowledge growth in teaching. Educational Researcher, 15(2), 4-14.

Türnüklü, E., Gündogdu Alayli, F. \& Akkas, E. N. (2013). Investigation of Prospective Primary Mathematics Teachers' Perceptions and Images for Quadrilaterals. Educational Sciences: Theory and Practice, 13(2), 1225-1232.

Van Hiele, P.M. (1986). Structure and insight. New York, NY: Academic Press.

Žilková, K., Gunčaga, J. \& Kopáčová, J. (2015). (Mis)Conceptions about geometric shapes in pre-service primary teachers. Acta Didactica Napocensia, 8(1), 27-35.

Žilková, K. (2013). Theory and Practice of Geometric Manipulations in Primary Education. Prague, Czech Republic: Powerprint. 